\title{
SEGURANÇA QUÍMICA E PREVENÇÃO DE INCÊNDIOS EM INSTITUIÇÕES DE ENSINO E PESQUISA QUE UTILIZAM PRODUTOS QUÍMICOS: UMA PROPOSTA DE NORMATIVA PARA O ESTADO DE SÃO PAULO.
}

\author{
Marcelo Higino A. Silveira ${ }^{1^{*}}$, Reinaldo C. Bazito ${ }^{2 *}$ \\ 1 - Corpo de Bombeiros da Polícia Militar do Estado de São Paulo, São Paulo, SP \\ 2 -Instituto de Química, Universidade de São Paulo (USP), São Paulo, SP \\ marcelohigino@uol.com.br
}

\begin{abstract}
Resumo: Um levantamento dos riscos inerentes aos materiais perigosos e da carga específica de incêndio no Instituto de Química da USP (IQUSP) mostrou a existência de situações preocupantes que demandavam correção. Não é um problema limitado ao IQUSP, haja vista que a maioria das instituições que oferecem cursos de graduação em Química no Estado de São Paulo, públicas e privadas, não possuem o Auto de Vistoria do Corpo de Bombeiros (AVCB), dentro das normas em vigor. Não há uma Instrução Técnica (IT) do Corpo de Bombeiros da Polícia Militar do Estado de São Paulo (CBPMESP) específica para prevenção de incêndios e acidentes com produtos químicos em instituições de ensino e pesquisa. Este trabalho descreve a proposta para o desenvolvimento dessa normativa, em sua etapa inicial, que envolve a adaptação à realidade brasileira da norma da National Fire Protection Association (NFPA), a NFPA 45 "Standard on Fire Protection for Laboratories Using Chemicals", utilizando o levantamento das condições atuais de algumas instituições de ensino e pesquisa que têm esse tipo de laboratório, em particular o IQUSP. Ao final do trabalho será proposta uma Instrução Técnica do CBPMESP adequada à esse tipo de instituição.
\end{abstract}

Palavras-chave: Segurança Química; Instrução Técnica; Prevenção de Incêndios; AVCB; Laboratórios de Ensino e Pesquisa.

\section{Chemical safety and fire prevention in teaching and research institutions using chemicals products, a case study in the São Paulo state}

Abstract: A survey of the risks inherent to hazardous materials and the specific fire load at the Institute of Chemistry of the University of São Paulo (IQUSP) showed the existence of worrying situations that demanded corrections. It is not a problem limited to IQUSP, considering that the majority of the institutions which offer chemistry undergraduate courses in the State of São Paulo, public or private, don't have the term of inspection of the fire department (AVCB), within the valid regulations. There is no Technical Instruction (IT) of the Fire Department of the Military Police of the State of São Paulo (CBPMESP) for the prevention of fire and accidents with chemicals for institutions of teaching and research. This work describes the proposal for the development of such a regulation, in its initial stage, that involves the adaptation to the Brazilian reality of the National Fire Protection Association (NFPA), the NFPA 45 "Standard on Fire Protection for Laboratories Using Chemicals", using the survey of the current conditions in some institutions of teaching and research that have this kind of laboratories, specially the IQUSP. A Technical Instruction of CBPMESP adequate to this kind of institution will be proposed at the end of this work.

Keywords: Chemical Safety; Technical Instruction; Fire Prevention; AVCB; Teaching and Research Laboratories.

\section{Introdução}

$\mathrm{O}$ armazenamento, uso ou descarte de produtos químicos perigosos em ambientes de trabalho ou estudo demandam medidas de segurança e prevenção de incêndios e de acidentes com esses produtos bem estabelecidas, de forma a reduzir os riscos e proteger a vida e a integridade física das pessoas, $o$ patrimônio e o meio ambiente. Normas de segurança bem definidas, capacitação adequada e uma cultura de segurança institucionalizada são essenciais para isso. ${ }^{1}$ Instituições de ensino e pesquisa em que esses produtos químicos perigosos estão presentes muitas vezes não têm uma cultura de segurança bem estabelecida, aumentando o risco de acidentes. ${ }^{2}$ No Brasil a situação não é diferente, com o agravante que muitas vezes essa cultura de segurança ainda muito incipiente aparece associada 
a instalações em edificações mais antigas, em desacordo com as normas modernas de prevenção de incêndios e acidentes com materiais perigosos.

A motivação para este trabalho surgiu de um extenso levantamento das condições de segurança visando a regularização de uma dessas instituições, o Instituto de Química da USP. ${ }^{3}$ Algumas das situações encontradas foram a grande carga de incêndio relacionada aos produtos químicos armazenados, a inadequação da comunicação de perigos/riscos e da armazenagem de parte desses materiais, que ocorria inclusive em corredores e rotas de fuga, a ausência de uma equipe treinada para $o$ atendimento a eventuais acidentes, incêndios e outras emergências, entre outras, além da cultura de segurança pouco arraigada. Percebeu-se também a inadequação das normas de prevenção de incêndio à situação específica dessas instituições, onde normalmente há uma grande variedade de produtos químicos perigosos, em embalagens individuais, sendo estocados ou manipulados nos laboratórios e almoxarifados.

Diante disso, propõe-se que o Corpo de Bombeiros da Polícia Militar do Estado de São Paulo (CBPMESP) elabore uma Instrução Técnica (IT) própria para regular a sinalização de emergência para perigos/riscos associados a produtos químicos, as condições de armazenagem adequadas desses produtos, dentro de outras normas existentes, para garantir condições mínimas de segurança para a comunidade da instituição e criar uma cultura de segurança, além de garantir às equipes de emergência a identificação de perigos/riscos existentes, em situações de atendimentos emergenciais. Essa IT específica para laboratórios de instituições de ensino e pesquisa que usam produtos químicos preencheria a lacuna hoje existente e será baseada em uma adaptação da norma americana NFPA $45^{4}$, entre outras, à realidade brasileira.

\section{Regulação Existente}

As edificações ocupadas por instituições de ensino e pesquisa no Estado de São Paulo devem seguir, assim como as demais, a legislação de prevenção contra incêndios, que prevê a emissão de um Auto de Vistoria do Corpo de Bombeiros (AVCB), atestando que, no momento da vistoria, as medidas de prevenção atendiam às exigências da legislação. ${ }^{5}$ Essas exigências são descritas nas Instruções Técnicas do CBPMESP, desenvolvidas com base em uma série de normas técnicas, entre elas as da National Fire Protection Association (NFPA). A NFPA é uma organização internacional autofinanciada sem fins lucrativos, com sede nos Estados Unidos, criada para promover a proteção e prevenção de incêndios, amplamente conhecida por suas normas técnicas (National Fire Codes), desenvolvidas por especialistas em segurança contra incêndios, através das quais são recomendadas práticas seguras para uma série de situações. ${ }^{6}$

Não existe, no momento, uma IT específica para laboratórios que utilizem ou armazenem produtos químicos perigosos, quer sejam de instituições de ensino e pesquisa, quer sejam do setor industrial. Para suprir essa lacuna e poder auxiliar as instituições de ensino e pesquisa a adotarem as medidas preventivas tão necessárias, o trabalho desenvolvido neste mestrado profissional pretende adaptar a norma específica para laboratórios que manipulem ou armazenem produtos químicos desenvolvida pela NFPA, entre outras. A norma específica é a NFPA 45 - "Standard on Fire Protection for Laboratories Using Chemicals" 2019 Edition. ${ }^{4}$ Ela deve ser aplicada, nos Estados Unidos, país sede da NFPA, em edificações de laboratórios, unidades de laboratório e áreas de trabalho de laboratório, 
incluindo instalações educacionais. Há algumas exceções onde a norma não se aplica, que são descritas a seguir.

"(1) Laboratórios para os quais se aplicam as seguintes condições:

(a) Unidades de laboratório que contêm menos do que ou igual a $4 \mathrm{~L}$ de líquido inflamável ou combustível;

(b) Unidades de laboratório que contêm menos de 2,2 $\mathrm{m}^{3}$ de gás inflamável, não incluindo o gás canalizado de baixa pressão instalado de acordo com a NFPA 54 - "National Fuel Gas Code" (Código Nacional de Gás Combustível), 2021 Edition.

(2) Plantas piloto.

(3) Laboratórios que manipulam apenas produtos químicos com uma classificação de risco de 0 ou 1 para todos os seguintes: saúde, inflamabilidade e instabilidade, conforme definido pela NFPA 704.

(4) Laboratórios que são instalados em plantas fabris.

(5) Instalações de testes incidentais.

(6) Laboratórios físicos, eletrônicos, de instrumentos, laser ou semelhantes que usam produtos químicos apenas para fins incidentais, como limpeza.

(7) Perigos associados a materiais radioativos, que são previstos na NFPA 801.

(8) Laboratórios que trabalham apenas com material explosivo, que seguem a NFPA 495 - "Explosive Materials Code" (Código de Materiais Explosivos), 2018 Edition.

(9) Uma área de trabalho de laboratório contendo um risco de explosão grande o suficiente para causar danos à propriedade fora da área de trabalho do laboratório ou ferimentos fora da área de trabalho do laboratório que requerem tratamento médico, além dos primeiros socorros."

Ela prevê uma classificação de risco dos laboratórios que determina as medidas de prevenção necessárias, que incluem armazenamento adequado aos perigos/riscos, ventilação do ambiente, sistemas de combate a incêndios, comunicação de perigos/riscos, entre outros. O trabalho aqui desenvolvido busca identificar a realidade das instituições de ensino e pesquisa no Brasil e adaptar a norma NFPA a essa realidade e à nossa legislação.

Uma das dificuldades é a própria forma de classificação de perigos/riscos e sua comunicação. A NFPA recomenda, em sua norma NFPA 45, que seja seguida a norma NFPA 704 para isso, que é um sistema de identificação de perigos/riscos conhecido como diagrama de Hommel, uma verificação visual rápida do perigo para a saúde (azul), inflamabilidade (vermelho), reatividade (amarelo) e riscos especiais (branco) que podem apresentar uma substância química. ${ }^{7}$ Esse não é o sistema obrigatório no Brasil, mas ele é largamente utilizado tanto pelo CBPMESP como por outras equipes de atendimento a incêndios e emergências, que precisarão se adaptar ao sistema obrigatório.

O sistema adotado no Brasil é o Sistema Globalmente Harmonizado de Classificação e Rotulagem de Produtos Químicos (GHS). Ele nasceu de um acordo internacional adotado em 1992, na Conferência das Nações Unidas sobre Meio Ambiente e Desenvolvimento (ECO-92). As recomendações para o GHS foram publicadas pela primeira vez em fevereiro de 2003, sendo a oitava edição a mais recente, publicada em 2019. ${ }^{8}$ A Associação Brasileira de Normas Técnicas (ABNT), que é o órgão oficial normativo, publicou a série de normas ABNT NBR 14725 (partes 1, 2, 3, 4), para normatizar o GHS no Brasil. ${ }^{9-12}$ Dessa forma, todos os produtos químicos (substâncias puras ou misturas) devem obrigatoriamente estar classificados, rotulados e providos de Ficha de Informação de Segurança de 
Produto Químico (FISPQ), de acordo com o GHS, exceto nos casos em que há legislação específica como, por exemplo, no caso dos produtos farmacêuticos em medicamentos.

No desenvolvimento da IT será adotado o GHS para a identificação de riscos químicos e de incêndio com produtos químicos. Isso incluirá a sinalização de risco/perigo nas instalações físicas dos laboratórios de pesquisa e ensino que trabalhem com produtos químicos, tanto pelo aspecto normativo, como pelo conhecimento dos pictogramas de risco/perigo definidos para a rotulagem, o que facilitará a rápida identificação das características desses produtos nas edificações. Isso é especialmente importante para as equipes de socorro externas, como o Corpo de Bombeiros, que venham a atender emergências, possibilitando a identificação dos riscos envolvidos para minimizar os danos que possam ser ocasionados. A adoção do GHS significará também uma redução nas variações entre os sistemas de classificação independentes (por exemplo da NFPA) e um movimento em direção à comunicação global de risco.

Atualmente o CBPMESP tem 45 Instruções Técnicas (IT), sendo a que trata de produtos perigosos a IT 32/2019 - Produtos Perigosos em Edificações e Áreas de Risco ${ }^{13}$. Ela estabelece, em complementação às demais ITs, alguns parâmetros para proteção em geral, porém não abrange a sinalização de risco específica. Adota ainda sinalização similar à de transporte, como obrigatória, facultando em complementação o uso da NFPA 7047, e prevendo o armazenamento respeitando a compatibilidade de produtos químicos e quantidades quando fracionados em sua estocagem e manipulação.

\section{Quadro Situacional}

O Brasil tem um histórico de acidentes com laboratórios de instituições de ensino e pesquisa, alguns dos quais descritos a seguir:

1989 - Incêndio em Laboratórios de Pesquisa do IQUSP, danos materiais; ${ }^{14}$

2009 - Incêndio no Instituto de Química da Universidade Federal da Bahia (UFBA), extensos danos materiais; ${ }^{15}$

2010 - Explosão seguida de princípio de incêndio em laboratório de Química da Universidade Federal de Minas Gerais (UFMG), Campus Pampulha, uma vítima com queimaduras e danos materiais, ${ }^{16}$

2012 - Explosão em laboratório do Instituto de Química da Universidade de Brasília (UnB), dois feridos e danos materiais; ${ }^{17}$

2013 - Incêndio em laboratório de pesquisa do Instituto de Ciências da Saúde da UFBA, danos materiais de grande monta; ${ }^{18}$

2016 - Princípio de incêndio em laboratório de pesquisa do Instituto de Química da Universidade Federal do Rio Grande do Sul (UFRGS), danos materiais; $;^{19}$

2016 - Incêndio e explosão em laboratório de pesquisa do Instituto de Ciências Exatas da Universidade Federal de Juiz de Fora (UFJF), danos materiais; ${ }^{20}$

2017 - Incêndio no laboratório de farmácia da Faculdade Maurício de Nassau (UniNassau) em Fortaleza-CE, tumulto entre os graduandos e danos materiais, ${ }^{21}$

2018 - Explosão em laboratório no prédio da Metalurgia da Coppe, na Universidade Federal do Rio de Janeiro (UFRJ), três vítimas, sendo uma com queimaduras e outras duas feridas por estilhaços, ${ }^{22}$ 2019 - Incêndio em laboratório de produtos naturais na Universidade de Franca (Unifran), em FrancaSP, severos danos no laboratório; ${ }^{23}$

2020 - Incêndio no Laboratório de Química da UFPB, danos materiais; ${ }^{24}$ 
Esses são apenas alguns exemplos, que foram noticiados pela imprensa. Há muitas outras ocorrências que acabam subnotificadas ou não são amplamente divulgadas. Isso mostra a necessidade não apenas de uma normativa adequada para tentar reduzir esse número de ocorrências, em complementação às normas e ITs existentes, mas também do desenvolvimento de uma cultura de segurança adequada.

Além disso, as instituições de ensino e pesquisa, muitas vezes, também não estão regularizadas de acordo com as normas vigentes. No Estado de São Paulo, um levantamento preliminar realizado nos arquivos do CBPMESP, mostrou que das 26 Instituições analisadas que possuem cursos de graduação em Química, apenas 9 têm AVCB válido de acordo com as ITs em vigor, as demais 17 não o possuem. O número de instituições sem AVCB deve ser ainda maior, pois não foram consideradas instituições que têm outros cursos com laboratórios que utilizam produtos químicos, tais como Engenharia Química, Farmácia etc.

\section{Conclusão}

Há a necessidade do desenvolvimento de norma adequada às condições brasileiras, na forma de uma Instrução Técnica do CBPMESP, de modo a melhorar as condições de segurança nos laboratórios que manipulam ou armazenam produtos químicos perigosos em instituições de ensino e pesquisa no Brasil e auxiliar na implantação de uma cultura de segurança adequada nessas instituições. É essencial que tais instituições façam essa adequação às normas existentes e que implementem uma cultura de segurança adequada, pois são as responsáveis pela formação dos futuros profissionais que atuarão manipulando produtos químicos perigosos.

\section{Agradecimentos}

Agradecemos ao apoio do Instituto de Química da USP e do Corpo de Bombeiros da Polícia do Estado de São Paulo.

\section{Referências}

1. Hudson, P. T. W.; Safety Management and Safety Culture: The Long, Hard and Winding Road. Em Occupational Health and Safety Management Systems. Proceedings of the First National Conference; Pearse, W. et al.; eds.; Crown Content: Melbourne, 2001, 3-32.

2. Schröder, I. et al.; J. Chem. Health Saf. 2016, 23(1), 12-23.

3. Bazito, R. C. et al.; Segurança contra incêndios e acidentes com materiais perigosos no IQ-USP. Universidade de São Paulo: São Paulo, 2016.

4. National Fire Protection Association; NFPA 45: Standard on Fire Protection for Laboratories Using Chemicals, NFPA: Quincy, 2019.

5. São Paulo; Decreto $N^{\circ}$ 63.911, de 10 de dezembro de 2018, Institui o Regulamento de Segurança contra Incêndio das edificações e áreas de risco no Estado de São Paulo e dá providências correlatas, 2018.

6. https://www.nfpa.org/About-NFPA, acessada em 10/11/2020.

7. National Fire Protection Association; NFPA 704: Standard System for the Identification of the Hazards of Materials for Emergency Response, NFPA: Quincy, 2017.

8. United Nations; Globally Harmonized System of Classification and Labelling of Chemicals (GHS) (ST/SG/AC.10/30/Rev.8), 8는 ed.; United Nations: New York, Geneva, 2019. 
9. Associação Brasileira de Normas Técnicas (ABNT); ABNT NBR 14725-1:2009- Produtos químicos - Informações sobre segurança, saúde e meio ambiente. Parte 1: Terminologia (versão corrigida de 26/01/2010), ABNT: Rio de Janeiro, 2009.

10. Associação Brasileira de Normas Técnicas (ABNT); ABNT NBR 14725-2:2019- Produtos químicos - Informações sobre segurança, saúde e meio ambiente. Parte 2: Sistema de classificação de perigo, ABNT: Rio de Janeiro, 2019.

11. Associação Brasileira de Normas Técnicas (ABNT); ABNT NBR 14725-3:2017- Produtos químicos - Informações sobre segurança, saúde e meio ambiente. Parte 3: Rotulagem, ABNT: Rio de Janeiro, 2017.

12. Associação Brasileira de Normas Técnicas (ABNT); ABNT NBR 14725-4:2014- Produtos químicos - Informações sobre segurança, saúde e meio ambiente. Parte 4: Ficha de informações de segurança de produtos químicos (FISPQ), ABNT: Rio de Janeiro, 2014.

13. Corpo de Bombeiros da Polícia Militar do Estado de São Paulo (CBPMESP); Instrução Técnica $N^{o}$ 32/2019 - Produtos Perigosos Em Edificações e Áreas de Risco; 2019.

14. Folha de São Paulo, Edição de 20/02/1989, Caderno Cidades, C4 (disponível em https://acervo.folha.com.br/) .

15. Setor Técnico Científico na Superintendência Regional na Bahia do Departamento de Polícia Federal, Laudo $\quad N^{\circ}$ 0526/2009 - SETEC/SR/DPF/BA (disponível em http://www.twiki.ufba.br/twiki/pub/Quimica/WebHome/2-LAUDO_INCENDIO.pdf, acesso em 10/11/2020)

16. https://www.ufmg.br/online/arquivos/017267.shtml, acesso em 10/11/2020.

17. http://g1.globo.com/distrito-federal/noticia/2012/10/explosao-em-laboratorio-de-quimica-daunb-deixa-dois-feridos.html, acesso em 10/11/2020.

18. http://g1.globo.com/bahia/noticia/2013/03/incendio-atinge-area-de-faculdade-da-ufba-dizpolicia-em-salvador.html, acesso em 10/11/2020.

19. http://www.ufrgs.br/ufrgs/noticias/esclarecimento-sobre-principio-de-incendio-em-laboratoriodo-instituto-de-quimica, acesso em 10/11/2020.

20. http://g1.globo.com/mg/zona-da-mata/noticia/2016/12/laboratorio-na-ufjf-e-isolado-aposincendio-e-explosao.html, acesso em 10/11/2020

21. https://diariodonordeste.verdesmares.com.br/metro/incendio-atinge-laboratorio-de-faculdadeem-fortaleza-1.1847410?page $=7$, acesso em 10/11/2020.

22. https://g1.globo.com/rj/rio-de-janeiro/noticia/2018/08/15/laboratorio-da-coppeufrj-registraexplosao.ghtml, acesso em 10/11/2020.

23. https://g1.globo.com/sp/ribeirao-preto-franca/noticia/2019/10/26/incendio-destroi-laboratoriode-produtos-naturais-na-unifran-em-franca-sp.ghtml, acesso em 10/11/2020. 